\title{
Different Epistemologies Beneath Similar Methods: The Case of Causal Loop Thinkers
}

\author{
Magoroh MARUYAMA \\ Aoyama Gakuin University, Tokyo
}

Causal loop thinkers are found scattered in several fields. Many of the researchers in the following three fields are causal loop thinkers: (1) General Systems Theories; (2) Operations Research and Management Science; (3) System Dynamics. Within each of these three clusters, there are subclusters. Epistemological differences exist among clusters as well as among subclusters. Conceptual cross-fertilization is rare but desirable. Treatment of heterogeneity varies from cluster to cluster and from a subcluster to another, but is absent among most of the causal loop thinkes.

Keywords: epistemological differences, general systems theories, operations research, management science, system dynamics, necessity and desirability of cultural and epistemological heterogeneity.

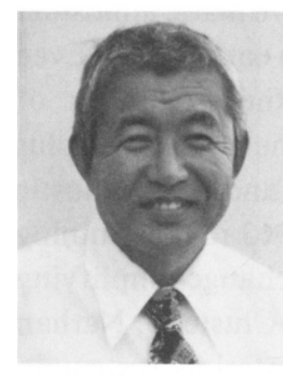

Magoroh Maruyama: born in Japan in 1929, studied at Universities of California (Berkeley), Munich, Heidelberg, Copenhagen and Lund. Taught at the Universities of California (Berkeley), Stanford, Illinois, Montpellier, Uppsala and Singapore. Author of 130 publications including The Second Cybernetics. Has been consultant to Volvo, NASA, Federal Motors of Indonesia, U.S. Dept. of Commerce, MITI of Japan, etc.
IOS Press

Human Systems Management 9 (1990) 195-198

\section{Introduction}

Causal loop thinkers working in different fields do not always exchange ideas, and often remain trapped in epistemologically limited versions of causal loops. The purpose of this article is to make the reader aware of the existence of various versions in order to promote cross-fertilization. It should not be interpreted as an attempt to classify the fields. In fact, in each field there are non-loop thinkers as well as many types of loop thinkers.

The concept of causal loops and interactive causality is many centuries old. In the organizational principles expressed in Japanese architecture and space composition, the concept can be traced back 5,000 years $[15,16]$. The development reached a high level of sophistication already 700 years ago. Explicit theoretical formulations occurred since 1910s [10, 20].

This article focuses on developments since 1950s. Beginning in 1950s, we have seen the emergence of General Systems Theories, Operations Research and Management Science, and System Dynamics. Many, but not all, in these fields are causal loop thinkers.

\section{Causal Loop Thinkers Among General Systems Theorists}

This cluster has at least three subclusters: (1a) Those whose primary interest is to find common 
principles, similarities and analogies among biological and social processes. They consider differences and heterogeneity to be non-essential, unimportant or uninteresting. Examples of thinkers are: Anatol Rapoport [24], James G. Miller (1b) Those who regard interacting heterogeneity as indispensable and desirable, and see evolution as a process of heterogenization as well as increase of interaction. Heterogeneity of epistemologies is also important. Examples of thinkers are: Maruyama, Jantsch, Morin, Camara, Prigogine. (1c) Practitioners who recognize and use epistemological types in business management, architecture, environmental design, extraterrestrial community design, employee selection and matching, etc. Examples of the practitioners are: Maruyama, Bechtel, Yatsuka.

There are marked individual differences among the thinkers in Subcluster 1b. Camara [1] and Maruyama [12, 13, 14, 15, 17, 18, 19] think in terms of interactions among members of a community or a group, and between humans and animals. For them the interactions are basically horizontal. Morin [22], on the other hand, thinks in terms of interactions between organizational levels, for example between the whole and the parts. For him the interactions are basically vertical. Jantsch $[7,8]$ in his lifetime was interested in self-organization but seldom talked about causal loops. Prigogine [23] is interactionist in his mathematics, but psychologically he keeps falling back on the concept of prime mover, as evidenced by his emphasis on and preoccupation with Big Bang, dissipation, exogenous energy input, bifurcation points and catastrophe theory in which changes can be generated at singular points, but not at other points whose settheoretical density is much higher than that of singular points. On the other hand Maruyama has pointed out $[9,11,14]$ the possibility that even without exogenous energy input, new patters may grow, and the amount of information may increase, because energy and entropy are two independent quantities and do not convert to and from each other [14]. Condensation Theory in astronomy is an example of such a possibility [11]. Furthermore, Prigogine considers Democritos as the forerunner of his concept. This indicates that his protoconcept is independent events, not interaction [15].

\section{Operations Research and Management Science}

This cluster is mainly interested in specifying the equations and coefficients of interaction, constructing mathematical models, testing the models against empirical data, and modifying the equations and coefficients to improve the fit between the models and the data. It is oriented toward trial and error, tinkering of models, and quantitative finetuning. Epistemological questions are of less importance.

\section{System Dynamics}

Richardson [25] lists 14 assumptions in economic theories, with which System Dynamics disagrees. Beyond them, there are two overall considerations which are repeatedly emphasized by authors in this cluster $[21,25,26,27]$. The first is that disequilibrium may result endogenously, contrary to the assumption in economics that disequilibrium is caused by exogenous factors. The second is that causal loops may produce unexpected results which cannot be anticipated by segment-by-segment analysis.

These two considerations are the same as what had been stated by those in Cluster $1 \mathrm{~b}[9,10,11]$. It is worth noting that Cluster 1 and Cluster 3 must have developed these concepts independently, as the authors of the two clusters have made almost no reference to each other's group in early 1960s. Even today Jay Forrester, who is the originator of Cluster 3, does not make use of the now classic plus and minus sign notation system and its diagnostic value devised by Maruyama in 1963 in determining whether given causal loops are change-amplifying or change-counteracting. Within Cluster 3, Nathan B. Forrester [6] adopted the notation system in 1973, and George Richardson [25] the same in 1983. However, the effect of time delay in the loop which may produce oscillation instead of stabilization in change-counteracting loops as discussed by Maruyama in 1963 ([10], page 177) has not yet been incorporated by Richardson $[25,26]$.

It appears that Cluster 3 has at least two subclusters: (3a) Those who work on endogenously induced changes, which asymptotically reach a new 
equilibrium, oscillate within a limit, or oscillate with decreasing amplitude. They do not use plus and minus signs on each segment of a causal loop, though they may put one sign to a loop as a whole. Examples are: Jay Forrester [2, 3, 4, 5] and Morecroft [21]. Another Subcluster (3b) consists of those who include the possibility of change amplification. They tend to use plus and minus signs on each segment of a causal loop. Examples are: $\mathrm{Na}-$ than B. Forrester [6], Richardson [25, 26], Wolstenholme and Coyle [27].

\section{Crossclusters}

In one respect, Subcluster $1 \mathrm{~b}$ and Subcluster $3 \mathrm{~b}$ share a common tendency in the sense that both consider change-amplifying loops. However, the two subclusters differ in a very basic respect. For the former, heterogeneity is a very basic concept, but for the latter it is not a central concept. There is undoubtedly another Subcluster 3c, whose main interest is model-tinkering and quantitative finetuning. Subcluster $3 \mathrm{c}$ would make a crosscluster with Cluster 2. The relative absence of quantitative fine-tuners and model thinkerers in Cluster 1 is a weakness of this cluster.

\section{The Concept of Heterogeneity}

The quantitative aspects of the behavior of causal loops are tangible and easily understood. On the other hand the qualitative aspects often remain unappreciated. Among them are the necessity and desirability of increase of heterogeneity and positive-sum interactions among heterogeneous elements in ecological, social and cultural systems. Even among those who consider heterogeneity, many are missing the point. The argument in terms of resource diversification is of a zero-sum type. That of risk dispersion is of a negative-sum type. Furthermore, while material heterogeneity is more easily understood because it can be quantified, the necessity and desirability of cultural and epistemological diversity are appreciated by only a very small number of thinkers. Maruyama [13, 14, 15] has elaborated on these points. Are most of the causal loop thinkers "materialists" in this sense? Or are they homogenists? If so, they are missing the important half of the meaning of causal loops.

\section{References}

[1] Camara, S. (1975) The Concept of Heterogeneity and Change among the Mandenka, Technological Forecasting and Social Change 7:273-284.

[2] Forrester, J.W. (1961) Industrial Dynamics. Cambridge: MIT Press.

[3] Forrester, J.W. (1968) Principles of Systems. Cambridge: MIT Press.

[4] Forrester, J.W. (1969) Urban Dynamics. Cambridge: MIT Press.

[5] Forrester, J.W. (1971) World Dynamics. Cambridge: MIT Press.

[6] Forrester, N.B. (1973) Life Cycle of Economic Development. Cambridge: Wright-Allen Press.

[7] Jantsch, E. (1976) Evolution and Consciousness. Reading: Addison-Wesley.

[8] Jantsch, E. (1980) Self-organizing Universe. New York: Pergamon.

[9] Maruyama, M. (1960) Morphogenesis and Morphostasis. Methodos 12:251-296.

[10] Maruyama, M. (1963a) The Second Cybernetics: Deviation-amplifying Mutual Causal Processes. American Scientist 51:164-179.

[11] Maruyama, M. (1963b) Postscript to the Second Cybernetics. American Scientist 51:250-256.

[12] Maruyama, M. (1974) Paradigmatology. Cybernetica $17: 136-156 ; 237-281$.

[13] Maruyama, M. (1977) Heterogenistics. Acta Biotheoretica 26:120-136.

[14] Maruyama, M. (1978) Heterogenistics and Morphogenetics. Theory and Society 5:75-96.

[15] Maruyama, M. (1980) Mindscapes and Science Theories. Current Anthropology 21:589-599.

[16] Maruyama, M. (1981) Denkmuster: Metaprinzipien der Umweltgestaltung. Garten und Landschaft Oktober 806-815.

[17] Maruyama, M. (1984) Alternative Concepts of Management: Insights from Asia and Africa. Asia Pacific Journal of Management 1:100-111.

[18] Maruyama, M. (1985) Mindscapes: How to Understand Specific Situations in Multicultural Management. Asia Pacific Journal of Management 2:125-149.

[19] Maruyama, M. (1989) Epistemological Sources of New Business Problems in the International Environment. $\mathrm{Hu}$ man Systems Management 8:71-80.

[20] Milsum, J. (1968) Positive Feedback. New York: Pergamon.

[21] Morecroft, J.D. (1985) Rationality in the Analysis of Behavioral Simulation Models. Management Science 31:900-916.

[22] Morin, E. (1977) La Méthode. Paris: Seuil. 
[23] Prigogine, I. and G. Nicholis (1977) Self-organization in Nonequilibrium Systems. New York: Wiley.

[24] Rapoport, A. (1983) Mathematical Models in the Social and Behavioral Sciences. Ann Arbor: University of Michian Press.

[25] Richardson, G.P. (1983) The Feedback Concept in American Social Science. Paper presented at 1983 International System Dynamics Conference.
[26] Richardson, G.P. (1989) System Dynamics: Simulating Feedback Systems for Policy Analysis. in P.K. Fishwick and P.A. Luker (Eds), Qualitative Simulation Modeling and Analysis. New York: Springer Verlag.

[27] Wolstenholme, E.F. and R.G. Coyle (1983) The Development of System Dynamics as a Methodology for System Description and Qualitative Analysis. Journal of Operations Research Society 34:569-581. 